IP Periodica Polytechnica

Mechanical Engineering

60(4), pp. 238-246, 2016

DOI: $10.3311 /$ PPme.8999

Creative Commons Attribution (i)

RESEARCH ARTICLE

\section{Parameter Reduction in the Frequency Analysis of Center of Pressure in Stabilometry}

\author{
Gergely Nagymáté ${ }^{*}$, Rita M. Kiss ${ }^{1}$
}

Received 10 January 2016; accepted after revision 23 June 2016

\begin{abstract}
During postural stability evaluation frequency analyses are applied on the motion of the foot center of pressure (CoP). In this study we compared the most widely used frequency type CoP parameters (median frequency, mean power frequency, bandwidth) including some newly defined parameters (frequency range power ratios, spectral power ratio between the anteroposterior and mediolateral directions, largest CoP amplitude with the corresponding frequency). The parameters were acquired from 25 young and healthy participants. Correlation analysis was used to exclude parameters that contain redundant information. Variance analysis was used to evaluate the behavior and usability of the parameters in different stance conditions (bipedal stance with eyes open and eyes closed, single leg stance with eyes open) where the balancing capability alters. Based on our results we recommend using the largest amplitude and frequency power ratios between specified frequency bands, spectral power ratio between anteroposterior and mediolateral direction and mean power frequency.
\end{abstract}

\section{Keywords}

stabilometry, center of pressure, frequency analysis

\section{Introduction}

Stabilometry is a method commonly used to assess postural stability during bipedal or/and single limb quiet standing by analyzing the displacement of the foot center of pressure (CoP). The CoP is often measured on force distribution plates. Several biomechanical observations and clinical diagnostics can be concluded from the statistical analysis of CoP displacement [1]. Numerous neural or physiological alterations significantly modify postural stability which alterations are reflected on CoP displacement. An instance of the neurological alterations mentioned is Parkinson's disease whose early diagnosis can be reportedly predicted based on $\mathrm{CoP}$ analysis [2]. CoP analysis can reveal biomechanical effects of physiological alterations such as discrepancies in the postural stability of patients with different degrees of knee osteoarthritis [3] or between bilateral or unilateral involvement [4].

Different stance types can be used for stabilometry measurements. Bipedal quiet stance is a common postural test case as it is a natural postural condition. The loss of visual information significantly alters the balancing capability [5, 6], therefore bilateral stance with eyes closed is also often used as a test condition by researchers to analyze postural differences, for example in rehabilitation monitoring [7]. Single leg stance also shows different postural characteristics from bipedal stances and it is rather suitable for analyzing ankle related conditions [8].

From the time varying coordinates of the CoP several parameters can be derived that can be classified into two large groups: distance type and frequency based parameters. The distance type parameters statistically describe the path and extension of the CoP trajectory. Typical distance type parameters are path length, 95\% confidence ellipse, maximum ranges and standard deviations of the instantaneous CoP points. Several similar parameters can be derived from these that can be found in the literature $[9,10]$; however, many of these parameters contain redundant information as shown by correlation analysis. Redundant parameters can be replaced by new parameters based on their combination with other parameters; for example, by the ratio of the same parameters in anteroposterior (AP) and mediolateral (ML) directions. These parameters can

\footnotetext{
${ }^{1}$ Department of Mechatronics, Optics and Mechanical Engineering, Informatics, Faculty of Mechanical Engineering, Budapest University of Technology and Economics, Müegyetem rakpart 3, Budapest 1111, Hungary

*Corresponding author, e-mail: nagymate@mogi.bme.hu
} 
analyze CoP displacement from a new point of view. To assess the responsiveness of these new parameters variance analysis can be performed on groups with knowingly different postural characteristics or in case of a relatively homogeneous group in different stance types as significant intrapersonal differences also occur between stance types [5, 6]. These differences are similar to those between healthy subjects and subjects with impaired balance.

On the basis of our research using correlation and variance analysis on distance type CoP parameters the following parameters are recommended: $95 \%$ confidence ellipse area, CoP path length, maximum CoP velocity, confidence ellipse axis ratio and AP-ML range ratio [10].

Non-stationarities of postural sway have already been reported as trends in CoP movement [11]. Numerous studies describe the variation of $\mathrm{CoP}$ frequency spectrum by time frequency analysis and visualization in spectrograms [12] and time-frequency graphs [13-15]. Chiaramello et al. provided a possible solution for the automatic processing of these spectrograms based on image processing algorithms [16]. CoP time-frequency analyses are often carried out by Short-time Fourier Transformation (STFT) [17], Wavelet transformation [18] or evolutionary spectral estimator [19]. Other frequency based analyses consider the frequency spectrum for the whole interval of stance trials. These studies analyze the frequency content of the whole measurement using Power Spectral Density (PSD) [12, 13, 20]. These studies often examine the median frequency (MDF) [21, 22], mean power frequency (MPF) $[15,20,23]$ and bandwidth (BW) $[15,23]$ of the CoP signals in AP and ML directions. Further analyses concern the power ratios of different frequency bands on the power spectrum of CoP signals, revealing differences in the frequency content of eyes closed and eyes open bilateral standing conditions in people with multiple sclerosis [24] or in athletes [5]. Many frequency-related posturographic parameters are highly sensitive to acquisition settings [25] and parameter settings of the different spectral estimators [21], with a resulting diversity in the results of the same measurements among different studies. Thus suggestions of standardized measurements and processing $[21,25]$ as well as minimal acquisition criteria have been reported [26, 27].

Other conditions such as acquisition time, foot position, and arm position are also important factors when the reliability of the CoP parameters is important. Ruhe et al. in [28] summarize the most widely applied procedures in a systematic literature review and suggest the best practice for the most reliable CoP measurements regarding experimental setup, sampling frequency and duration, visual conditions and foot positions. In 2013 the International Society for Posture and Gait Research (I.S.P.G.R.) published several standardization statements regarding these issues [26] among which many have been applied as detailed in the following section.
The diversity of the frequency based parameters even with the standardized minimal acquisition and processing settings offer a large applicable parameter set. Certain combinations of the possible parameters lead to reportedly reliable conclusions. However, reduction in the number of frequency based parameters may be beneficial to approach a more standardized measurement form. The first goal of the present study is to reduce the number of the frequency based $\mathrm{CoP}$ parameters by excluding those that contain redundant information by showing strong correlation to other parameters. The second goal is to introduce new expressive parameters for analyzing balancing capability in different postural conditions, as a replacement for the excluded parameters.

\section{Methods \\ 2.1 Participants}

25 young healthy individuals were involved in the study (22 males, 3 females: $22.34 \pm 0.97$ years, $74.34 \pm 4.23 \mathrm{~kg}, 1.711$ $\pm 0.085 \mathrm{~m}$, BMI: $25.3 \pm 2.8$ ). As the main interest was on the within-subject effect of the different stance types by repeated measures, participants were not separated by gender to achieve higher sample homogeneity. The participants did not suffer any musculoskeletal injury and did not go through surgery in the last ten years. The participants were informed in writing about the risks and benefits of the study; each gave signed informed consent and was given the opportunity to withdraw from the study at any time. The study was approved by the Hungarian National Science and Research Ethics Committee (114/2004).

\subsection{Experimental procedure}

A Zebris multifunctional force distribution measuring plate (type: Force Distribution Measurement-Small FDM-S, 320mm x 470mm measuring surface with 1504 load cells, ZEBRIS $\mathrm{GmbH}$, Isny, Germany) was used to collect force distribution data during standing. Accuracy of this force measuring plate was reported within $5 \%$ by Braun et al. [29]. In respect to the experimental protocol (acquisition time, foot and arm position) standardization suggestions from both Ruhe et al. [28] and the I.S.P.G.R [26] have been considered. Every participant performed three 60 second long barefoot trials successively in bipedal stance eyes open (EO) and eyes closed (EC) conditions (Fig. 1) and an additional 60 seconds long single leg stance (SL) with eyes open. Single leg eyes closed trial was skipped because the increased risk of falling and therefore lower feasibility. Sudden stand up may cause about 15 seconds of dizziness caused by blood pressure drop [30]. To avoid this affecting the trials participants were asked to stand up when the previous participant finished the first trial. Therefore the participants were standing about 2 minutes before their trial started. 


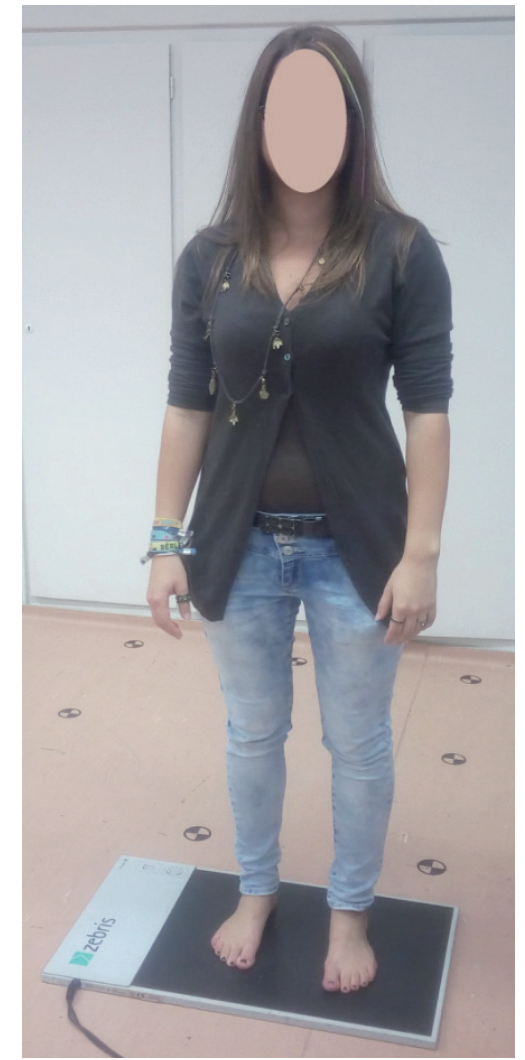

Fig. 1 Bipedal stance on force distribution plate

They were asked to look straight ahead (but not at any particular object) and have the arms at the sides, possibly loading both legs equally during the bipedal trials in a shoulder width stance, while standing on the dominant leg during the SL trial.

\subsection{Data processing}

Force distribution was recorded using the Zebris WinPDMS processing software v1.2 (zebris GmbH, Isny Germany). This software runs only on a Microsoft ${ }^{\circledR}$ Windows ${ }^{\circledR}$ XP ${ }^{\circledR} 32$ bit operation system. The sampling frequency was $30 \mathrm{~Hz}$ which satisfies the Shannon sampling theory [31], considering that $90 \%$ of the spectral power is under $2 \mathrm{~Hz}$ [15] and the frequency content of the CoP signal above $5-10 \mathrm{~Hz}$ is considered noise as it is often low-pass filtered [14, 15, 32].

Raw force distribution data was obtained from the WinPDMS software and was processed in custom processing software written in LabVIEW 2013 (National Instruments Inc., Austin, Texas). This software extracts instantaneous CoP coordinates from the force distribution data and calculates the frequency analysis data from it in AP and ML directions. From the CoP position signals power spectrum was obtained using Fast Fourier Transformation (FFT) with Hanning window. The acquired frequency resolution was $0.016 \mathrm{~Hz}$.

\subsection{Calculated parameters}

This section introduces the calculated parameters, firstly those that can be found in the literature.

Mean power frequency (MPF) is a weighted average frequency where $f_{j}$ frequency components are weighted by their $P_{j}$ power. $M$ is the number of frequency bins. MPF if calculated as proposed by Oskoei and $\mathrm{Hu}[33]$ :

$$
M P F=\sum_{j=1}^{M} f_{j} P_{j} / \sum_{j=1}^{M} P_{j}
$$

Median frequency (MDF): the frequency value which separates the power spectrum into two equal energy areas. MDF was calculated as proposed by Oskoei and $\mathrm{Hu}[33]$ :

$$
\sum_{j=1}^{M D F} P_{j}=\sum_{j=M D F}^{M} P_{j}=\frac{1}{2} \sum_{j=1}^{M} P_{j}
$$

99\% Bandwidth (BW) is the width of the frequency band in $\mathrm{Hz}$ in which $99 \%$ of the total power of the CoP signal is located.

The following parameters are newly defined explicitly in this paper, however the content of the first two have already been analyzed by other researchers.

Frequency range power ratios (LMR, MHR) are the parameterized values of the power distribution between the low- $(0-0.3 \mathrm{~Hz})$ medium- $(0.3-1 \mathrm{~Hz})$ and high frequency (1-5 Hz) bands defined by low-medium frequency range power ratio (LMR) and medium-high frequency range power ratio (MHR) in AP and ML directions. The dependencies of these frequency bands were studied separately by Nagy et al. [5]; however, they specified the upper limit of the high frequency band at $3 \mathrm{~Hz}$. The $99 \%$ bandwidth measurements revealed that in some cases the bandwidth of the CoP signal is wider than $3 \mathrm{~Hz}$, therefore we set the high frequency band upper limit to $5 \mathrm{~Hz}$. However, significant differences in the results did not occur between the two settings.

Spectral Power AP-ML Ratio (SPR) is the ratio of the total spectral power in AP direction and the total spectral power in ML direction. SPR characterizes the rate of power distribution of postural sway frequencies in the AP-ML directions.

Largest amplitudes (LA) and corresponding largest amplitude frequencies (LAF) are calculated from the timedomain of the CoP signal in AP and ML directions. Peaks and valleys of the signal are located and the largest peak-valley or valley-peak transition in amplitude is considered the double of the amplitude of the modeled sine function. The temporal difference between the selected signal peak and valley is the half period of the sine function. From these variables of the modeled sine function the LA and LAF are calculated (Fig. 2). 
Time-frequency parameters such as instantaneous MDF, -MPF and -BW were not calculated as they are instantaneous parameters and cannot characterize postural stability with only one number for the whole trial, but just for a time instance. In this study we are looking for parameter combinations that can describe one's balancing capabilities by a small set of numbers.

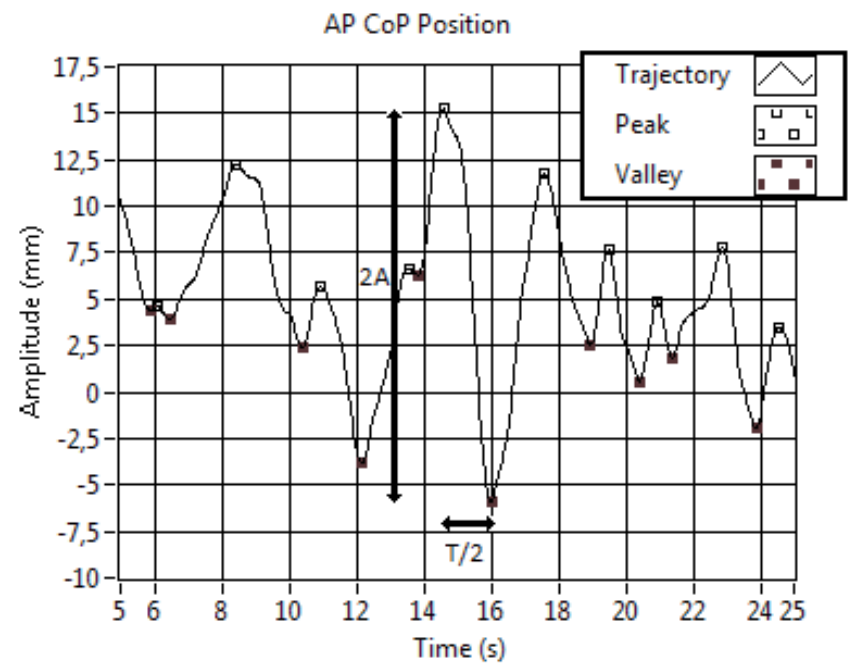

Fig. 2 Time domain determination of the largest amplitude and the corresponding frequency

\subsection{Statistical analysis}

The combination redundancies of the previously used (MPF, MDF, BW) and newly defined (LMR, MHR, SPR, LA, LAF) frequency-based $\mathrm{CoP}$ parameters were studied by correlation analysis. By excluding parameters that are strongly correlated to others, reduction in the parameter set can be achieved while keeping the diverse information gain from the different calculated parameters. Strong correlation was considered above 0.8 as suggested by Chan in [34], but moderate correlations above 0.5 were also taken into account.

The behavior of the previously used and new parameters was studied by variance analysis (ANOVA) for repeated measures. The alteration of postural stability thus $\mathrm{CoP}$ movement between different stance types (EO, EC, SL) have already been reported many times, therefore it is expected that the studied parameters indicate differences between the three stance types within subjects. Univariate test of ANOVA can tell whether there are statistically significant differences between the three stance types in respect to each parameter individually. Pairwise comparisons also indicate among which stance types the difference occurred. ANOVA requires meeting certain criteria of the studied variables, such as normal distribution, sphericity and extremum/outlier removal. The ensuring of these criteria and possible compensations are described in detail in [10]. During the statistical analysis the level of significance was $0.05(\alpha=0.05)$.

\section{Results}

\subsection{Correlation analysis}

The results of correlation analysis are summarized in Table 1. The strongest correlations can be observed in anteroposterior direction (AP) between the mean power frequency (MPF) and median frequency (MDF) (0.846).

Furthermore strong correlation can be observed between MPF and bandwidth (BW) in both mediolateral direction (ML) (0.812) and AP direction (0.767). It is interesting to note that there are several moderate negative correlations. In AP direction MPF negatively correlates with LMR (-0.613). Most correlations with other parameters are produced by $\mathrm{BW}$. BW in AP direction (BWAP) (besides MPF-AP) is moderately correlated with LAF- AP (0.504) and LA-ML (0.605). In AP direction BW negatively correlates with low-medium frequency range power ratio (LMR) $(-0.647)$ and medium-high frequency range power ratio (MHR) (-0.65). BW in AP direction also negatively correlates with MHR in ML direction (-0.603). In ML direction BW also negatively correlates with LMR (-0.557) and MHR (-0.583) and positively correlates with MDF (0.528). BWs in AP and in ML direction are also moderately correlated with each other (0.514). MHR correlates between AP and ML directions (0.642).

\subsection{Variance analysis}

The ANOVA addresses the investigation of the potential behavior of frequency-based COP parameters between stance types. To meet the ANOVA criteria described in [10], seven further participants had to be excluded from the study because some of their parameters appeared to be extremes compared to the rest of the cases especially in the ratio type parameters (Fig. 3). The final number of samples used to the repeated measures ANOVA was 18.

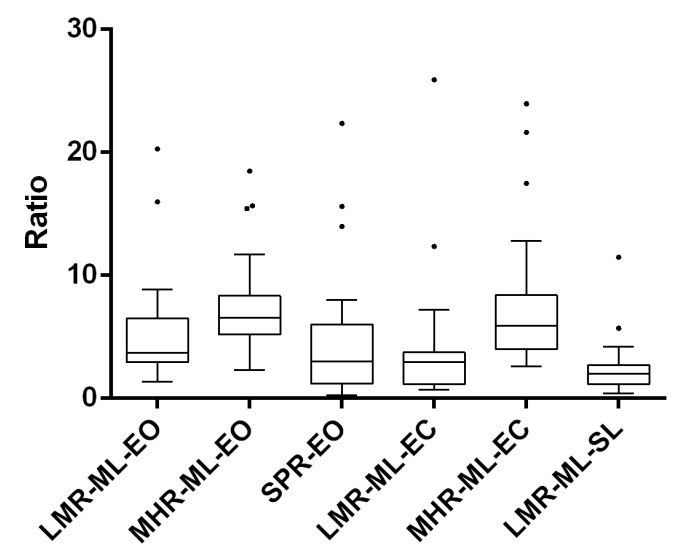

Fig. 3 Box plots with extreme values and outliers (dots) in some of the ratio type parameters. The data series of the dotted values are excluded to meet ANOVA criteria. LMR: low-medium frequency range power ratio, MHR: medium-high frequency range power ratio, SPR: spectral power ratio between the anteroposterior and mediolateral directions, ML: mediolateral direction, EO: bipedal stance with eyes open, EC: bipedal stance with eyes closed, SL: single leg stance with eyes open 
Table 1 Correlations

\begin{tabular}{|c|c|c|c|c|c|c|c|c|c|c|c|c|c|c|c|}
\hline & $\begin{array}{l}\text { LA- } \\
\text { AP }\end{array}$ & $\begin{array}{l}\text { LAF- } \\
\text { AP }\end{array}$ & $\begin{array}{l}\text { LA- } \\
\text { ML }\end{array}$ & $\begin{array}{l}\text { LAF- } \\
\text { ML }\end{array}$ & $\begin{array}{l}\text { LMR- } \\
\text { AP }\end{array}$ & $\begin{array}{l}\text { MHR- } \\
\text { AP }\end{array}$ & $\begin{array}{l}\text { LMR- } \\
\text { ML }\end{array}$ & $\begin{array}{l}\text { MHR- } \\
\text { ML }\end{array}$ & $\begin{array}{l}\text { MDF- } \\
\text { ML }\end{array}$ & $\begin{array}{l}\text { MDF- } \\
\text { AP }\end{array}$ & SPR & $\begin{array}{l}\text { BW- } \\
\text { AP }\end{array}$ & $\begin{array}{l}\text { BW- } \\
\text { ML }\end{array}$ & $\begin{array}{l}\text { MPF- } \\
\text { AP }\end{array}$ & $\begin{array}{l}\text { MPF- } \\
\text { ML }\end{array}$ \\
\hline LA-AP & 1 & & & & -0.199 & 0.009 & & 0.025 & 75 & -0.044 & -0.036 & 223 & .006 & 0.033 & .176 \\
\hline LAF & 37 & 1 & 0.367 & 4 & -0.286 & 77 & 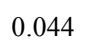 & 16 & 25 & 1 & 73 & 504 & 4 & 84 & 60 \\
\hline LA- & 499 & 26. & 1 & 0.19 & -0.345 & .372 & -0.072 & -0.306 & 286 & 057 & -0.479 & 605 & 118 & .282 & 0.221 \\
\hline LAF-ML & -0.098 & 0.284 & & 1 & -0.137 & -0.387 & -0.033 & -0.397 & 0.245 & & -0.100 & 0.414 & .352 & .302 & .156 \\
\hline LMR-A & .199 & -0.286 & -0.345 & .137 & 1 & 137 & 0.151 & 0.274 & .202 & -0.361 & 0.482 & -0.647 & -0.223 & -0.613 & 0.023 \\
\hline MHR-AI & 000 & 377 & -0.372 & .387 & 0.137 & 1 & -0.064 & 0.642 & -0.340 & -0.100 & 0.237 & -0.650 & -0.339 & -0.357 & .083 \\
\hline LMR-ML & .037 & 0.044 & -0.072 & -0.033 & 0.151 & -0.064 & 1 & -0.021 & -0.430 & -0.083 & -0.220 & -0.188 & -0.557 & -0.186 & -0.481 \\
\hline MHR-ML & 025 & -0.216 & -0.306 & -0.397 & 0.274 & 0.642 & -0.021 & 1 & -0.243 & -0.143 & 0.227 & -0.603 & -0.583 & -0.378 & -0.272 \\
\hline MDF-ML & 178 & 0.223 & 0.286 & 0.245 & -0.202 & -0.340 & -0.430 & -0.243 & 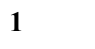 & 0.425 & 0.004 & 0.481 & 0.528 & 0.499 & 0.495 \\
\hline MDF-AP & .044 & 0323 & 0.057 & 20 & -0.361 & -0.100 & -0.083 & -0.143 & 0.425 & 1 & -0.164 & 0.389 & 0.235 & 0.846 & 0.287 \\
\hline SPR & -0.036 & -0.373 & -0.479 & -0.100 & 0.482 & 0.237 & -0.220 & 0.227 & 0.004 & -0.164 & 1 & -0.492 & 0.180 & -0.358 & 0.445 \\
\hline $\mathrm{BW}-\mathrm{AF}$ & 223 & 0.504 & 0.605 & 041 & -0.647 & -0.650 & -0.188 & -0.603 & ( 180 & 0.389 & -0.492 & 1 & 0.514 & 0.767 & 0.168 \\
\hline BW-ML & -0.006 & 0.104 & 0.118 & 0.352 & -0.223 & -0.339 & -0.557 & -0.583 & 0.528 & 0.235 & 0.180 & 0.514 & 1 & 0.429 & 0.812 \\
\hline MPF-AP & 0.033 & 0.484 & 0.282 & 0.302 & -0.613 & -0.357 & -0.186 & -0.378 & 0.499 & 0.846 & -0.358 & 0.767 & 0.429 & 1 & 0.319 \\
\hline MPF-ML & -0.176 & -0.060 & -0.221 & 0.156 & -0.023 & -0.083 & -0.481 & -0.272 & 0.495 & 0.287 & 0.445 & 0.168 & 0.812 & 0.319 & 1 \\
\hline
\end{tabular}

LA: Largest amplitude, LAF: Largest amplitude frequency, LMR: Low-medium frequency range power ratio, MHR: Medium-high frequency range power ratio, MDF median frequency, SPR: Spectral power ratio, BW: Bandwidth, MPF: mean power frequency, AP: anteroposterior direction, ML: mediolateral direction

Bold: $\mid$ correlation $\mid>=0.8$, Italic: $0.8>\mid$ correlation $\mid>=0.5$

Multivariate test showed a significant difference between the three stance types $(\mathrm{EO}, \mathrm{EC}, \mathrm{SL})(\mathrm{p}<0.001)$ as expected. Mauchly's test of sphericity [10] was only violated in two parameters (ML directional largest amplitude and median frequency) that the robust ANOVA could still handle. On the basis of the within-subject univariate test, all parameters except MDF-AP are significantly influenced by stance types, as they significantly differ from each other in the three stance types. As regards pairwise comparisons (Table 2), in respect of some parameters (LA-AP, LMR-ML, MDF-ML, MPF-ML) bipedal stance with EO significantly differ from both bipedal stance with EC and SL stance, while the latter two stance types do not differ significantly from each other. In the most common cases (LAF-AP, LA-ML, MHR-AP, MHR-ML, SPR, BW-AP, MPF-AP) EO and EC stances do not differ significantly from each other while SL stance statistically significantly differs from both bipedal stances. In case of some other parameters (LAF-ML, BW-ML) all the three stance types significantly differ from each other.

\section{Discussion}

Normally, stabilometry measurement results depend on age [6], physical fitness [5], previous injury [8], and any musculoskeletal disorder [9]. In this aspect our sample was homogeneous, although intra-personal changes were evaluated between the different stance conditions.
It is worth discussing the result of correlation analysis along with the ANOVA results. In most cases correlating parameters show similar behaviors to each other between stance types. Both BW-AP and BW-ML show a positive or negative correlation with many other parameters. BW-AP and most parameters correlating with it significantly differ between bipedal stance types and single leg stance. Exceptions are LMR-AP and BW-ML. BW-ML also moderately correlates with four parameters and strongly with MPF-ML. However, in case of BW-ML the differences between stance types developed less systematically. CoP bandwidth contains common information with many other parameters, but does not precisely express postural characteristics, therefore BW parameters are recommended for exclusion.

MHR moderately correlate to themselves between AP and ML directions. This might be due to the rotational characteristic of CoP path presumed by Chiaramello et al. [17]. In EC condition the CoP trajectory often becomes elongated as AP movements increase, thus the AP-ML range ratio increases [10]. The same behavior appears in the AP-ML spectral power ratio (SPR) of the CoP as oscillations are concentrated in the AP direction under EC condition (Fig. 4). In SL condition the SPR becomes smaller than in the bipedal stances as the oscillations are more evenly distributed in AP and ML directions. 
Table 2 Pairwise comparisons

\begin{tabular}{|c|c|c|c|c|c|c|c|}
\hline Measure & & & Mean Difference & Standard error & Level of significance & 95\% CI Lower Bound & 95\% CI Upper Bound \\
\hline \multirow[t]{3}{*}{ LA AP } & EO & $\mathrm{EC}$ & -5.563 & 1.601 & 0.003 & -8.940 & -2.185 \\
\hline & EO & SL & -7.263 & 1.654 & 0.000 & -10.752 & -3.774 \\
\hline & $\mathrm{EC}$ & SL & -1.700 & 1.814 & 0.362 & -5.528 & 2.127 \\
\hline \multirow[t]{3}{*}{ LAF AP } & $\mathrm{EO}$ & $\mathrm{EC}$ & 0.008 & 0.042 & 0.848 & -0.081 & 0.098 \\
\hline & $\mathrm{EO}$ & SL & -0.168 & 0.060 & 0.012 & -0.293 & -0.042 \\
\hline & $\mathrm{EC}$ & SL & -0.176 & 0.062 & 0.011 & -0.306 & -0.045 \\
\hline \multirow[t]{3}{*}{ LA ML } & EO & $\mathrm{EC}$ & -0.069 & 0.860 & 0.937 & -1.884 & 1.746 \\
\hline & $\mathrm{EO}$ & SL & -11.085 & 1.565 & 0.000 & -14.387 & -7.783 \\
\hline & $\mathrm{EC}$ & SL & -11.016 & 1.588 & 0.000 & -14.367 & -7.665 \\
\hline \multirow[t]{3}{*}{ LAF ML } & EO & $\mathrm{EC}$ & -0.095 & 0.044 & 0.044 & -0.187 & -0.003 \\
\hline & $\mathrm{EO}$ & SL & -0.254 & 0.054 & 0.000 & -0.367 & -0.140 \\
\hline & $\mathrm{EC}$ & SL & -0.158 & 0.048 & 0.004 & -0.259 & -0.058 \\
\hline \multirow[t]{3}{*}{ LMR AP } & $\mathrm{EO}$ & $\mathrm{EC}$ & 3.704 & 2.012 & 0.083 & -0.540 & 7.949 \\
\hline & $\mathrm{EO}$ & SL & 5.572 & 1.576 & 0.003 & 2.248 & 8.896 \\
\hline & $\mathrm{EC}$ & SL & 1.868 & 1.126 & 0.116 & -0.509 & 4.244 \\
\hline \multirow[t]{3}{*}{ MHR AP } & EO & $\mathrm{EC}$ & -0.829 & 0.892 & 0.366 & -2.711 & 1.053 \\
\hline & EO & SL & 3.288 & 0.895 & 0.002 & 1.400 & 5.176 \\
\hline & $\mathrm{EC}$ & SL & 4.117 & 1.030 & 0.001 & 1.943 & 6.291 \\
\hline \multirow[t]{3}{*}{ LMR ML } & EO & $\mathrm{EC}$ & 2.550 & 0.929 & 0.014 & 0.590 & 4.511 \\
\hline & $\mathrm{EO}$ & SL & 3.163 & 0.829 & 0.001 & 1.415 & 4.912 \\
\hline & $\mathrm{EC}$ & SL & 0.613 & 0.638 & 0.350 & -0.734 & 1.960 \\
\hline \multirow[t]{3}{*}{ MHR ML } & $\mathrm{EO}$ & $\mathrm{EC}$ & -0.905 & 1.082 & 0.415 & -3.186 & 1.377 \\
\hline & EO & SL & 4.673 & 0.906 & 0.000 & 2.761 & 6.585 \\
\hline & $\mathrm{EC}$ & SL & 5.578 & 1.301 & 0.000 & 2.833 & 8.322 \\
\hline \multirow[t]{3}{*}{ MDF ML } & EO & $\mathrm{EC}$ & -0.077 & 0.037 & 0.050 & -0.154 & 0.001 \\
\hline & EO & SL & -0.195 & 0.053 & 0.002 & -0.308 & -0.083 \\
\hline & $\mathrm{EC}$ & SL & -0.119 & 0.067 & 0.095 & -0.260 & 0.023 \\
\hline \multirow[t]{3}{*}{ MDF AP } & EO & $\mathrm{EC}$ & -0.029 & 0.017 & 0.110 & -0.065 & 0.007 \\
\hline & $\mathrm{EO}$ & SL & -0.006 & 0.015 & 0.662 & -0.037 & 0.024 \\
\hline & $\mathrm{EC}$ & SL & 0.022 & 0.021 & 0.307 & -0.022 & 0.067 \\
\hline \multirow[t]{3}{*}{ SPR } & $\mathrm{EO}$ & $\mathrm{EC}$ & -3.225 & 1.617 & 0.062 & -6.637 & 0.186 \\
\hline & EO & SL & 2.985 & 1.011 & 0.009 & 0.853 & 5.117 \\
\hline & $\mathrm{EC}$ & SL & 6.210 & 1.493 & 0.001 & 3.061 & 9.359 \\
\hline \multirow[t]{3}{*}{ BW AP } & EO & $\mathrm{EC}$ & -0.134 & 0.144 & 0.363 & -0.437 & 0.169 \\
\hline & $\mathrm{EO}$ & SL & -1.350 & 0.154 & 0.000 & -1.675 & -1.026 \\
\hline & $\mathrm{EC}$ & SL & -1.216 & 0.144 & 0.000 & -1.519 & -0.913 \\
\hline \multirow[t]{3}{*}{ BW ML } & $\mathrm{EO}$ & $\mathrm{EC}$ & -0.550 & 0.198 & 0.013 & -0.967 & -0.133 \\
\hline & $\mathrm{EO}$ & SL & -1.111 & 0.179 & 0.000 & -1.489 & -0.733 \\
\hline & $\mathrm{EC}$ & SL & -0.560 & 0.224 & 0.023 & -1.034 & -0.087 \\
\hline \multirow[t]{3}{*}{ MPF AP } & $\mathrm{EO}$ & $\mathrm{EC}$ & -0.037 & 0.027 & 0.188 & -0.094 & 0.020 \\
\hline & $\mathrm{EO}$ & SL & -0.148 & 0.033 & 0.000 & -0.219 & -0.078 \\
\hline & $\mathrm{EC}$ & SL & -0.111 & 0.035 & 0.006 & -0.186 & -0.037 \\
\hline \multirow[t]{3}{*}{ MPF ML } & $\mathrm{EO}$ & $\mathrm{EC}$ & -0.187 & 0.069 & 0.015 & -0.332 & -0.041 \\
\hline & $\mathrm{EO}$ & SL & -0.163 & 0.068 & 0.028 & -0.307 & -0.020 \\
\hline & $\mathrm{EC}$ & SL & 0.023 & 0.093 & 0.807 & -0.174 & 0.220 \\
\hline
\end{tabular}

CI: Confidence interval, AP: antero-posterior, ML: medio-lateral, LA: largest amplitude, LAF largest amplitude frequency, LMR: low-medium frequency range power ratio, MHR: medium-high frequency range power ratio, MDF: median frequency, SPR: spectral power ratio, BW: bandwidth, MPF: mean power frequency

Bold: significant difference $(\mathrm{p}<0.05)$ 


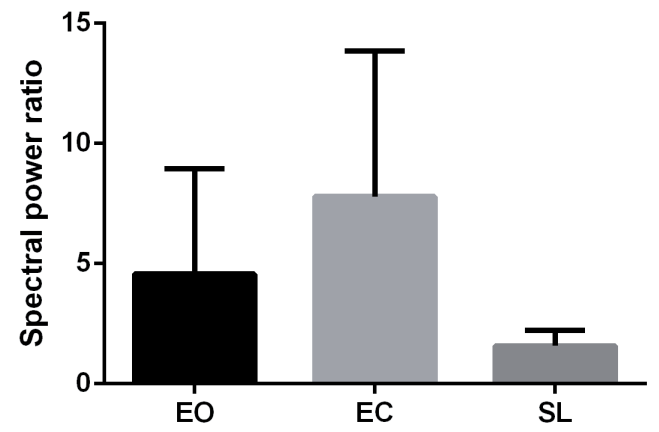

Fig. 4 Spectral power ratio between stance types (EO: eyes open bipedal, EC: eyes closed bipedal, SL: eyes open single leg stance)

The negative correlations between BW and the frequency band ratios (LMR and MHR) are reasonable as most of the frequency power shifted to the lower frequency bands the bandwidth narrows. A moderate negative correlation between MPF and LMR in AP direction has a similar explanation as MPF follows the high power frequencies.

MPF-AP and MDF-AP have such a strong correlation that only one of them should be sufficient to express the same phenomenon. It is interesting to note that in ML direction this correlation is not reflected. In the ANOVA results MDF-AP did not show any difference between stance types but MPF did in both directions. As MPF is also strongly correlated to BW in both directions and BW has already been excluded, preserving MPF and excluding MDF is recommended in the reduction of parameter number.

The newly defined LA and LAF parameters were graphically analyzed as well. Scatter plots and convex hulls around the LALAF points for each stance type are presented in Fig. 5. A and B. The frequency of the largest amplitude showed no correlation with the largest amplitude frequency (Table 1). As it can be seen on Fig. 5/A the LAF-AP values scatter uniformly approximately between 0.1 and $0.8 \mathrm{~Hz}$ in AP direction irrespective to the size of the amplitude. In ML direction (Fig. 5/B) the same applies to LAF in the approximate range from 0.1 to $0.9 \mathrm{~Hz}$. In ML direction the LA parameter clearly shows a separation of SL stance from bipedal stances. Therefore LA is appropriate parameter to characterize different stance types and possibly impairment conditions as well. LAF does not tell trustworthy information on postural stability, therefore its usage is not recommended.

The preserved CoP parameters can be useful in clinical cases. LA shows the amplitude of the continuous sway which was unobservable using conventional distance type CoP parameters - such as ranges and summary measures - as 95\% confidence ellipse sizes [10]. This largest amplitude describes more informatively the random errors of one's postural control than the summary measures and absolute ranges. LMR and MHR can numerically describe the deviations of frequency bands that were analyzed by Nagy et al. in [5]. These ratios may also reveal alterations in the frequency domain that was analyzed by timefrequency analysis before, e.g. on vestibularly impaired patients [14]. SPR could be useful in cases when the full spectral powers are separately analyzed in AP and ML directions. Oliveira et al in [35] have reported changes on the spectral power distribution of pregnant women that could be described by SPR. MPF is already a widely used CoP spectral parameter that is used in a variety of studies, e.g. to monitor the balance development of children [36], or to determine falling related balance problems of people with Parkinson's disease [37].

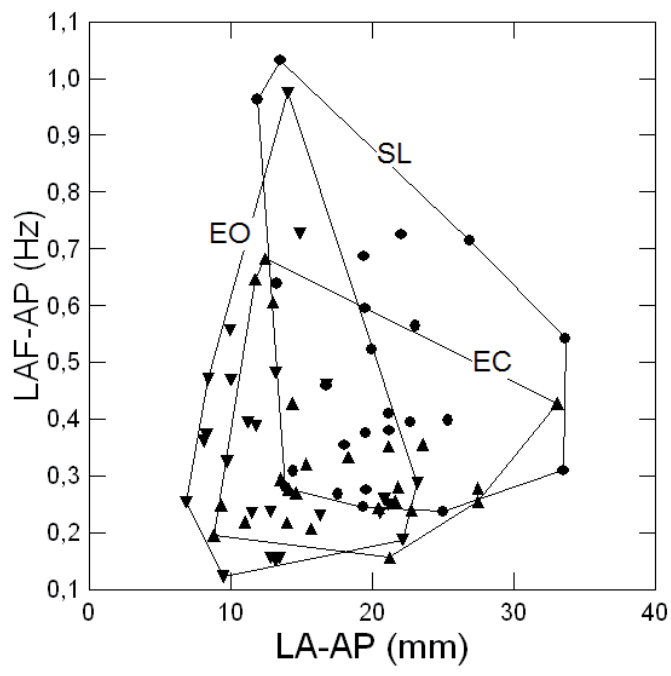

A

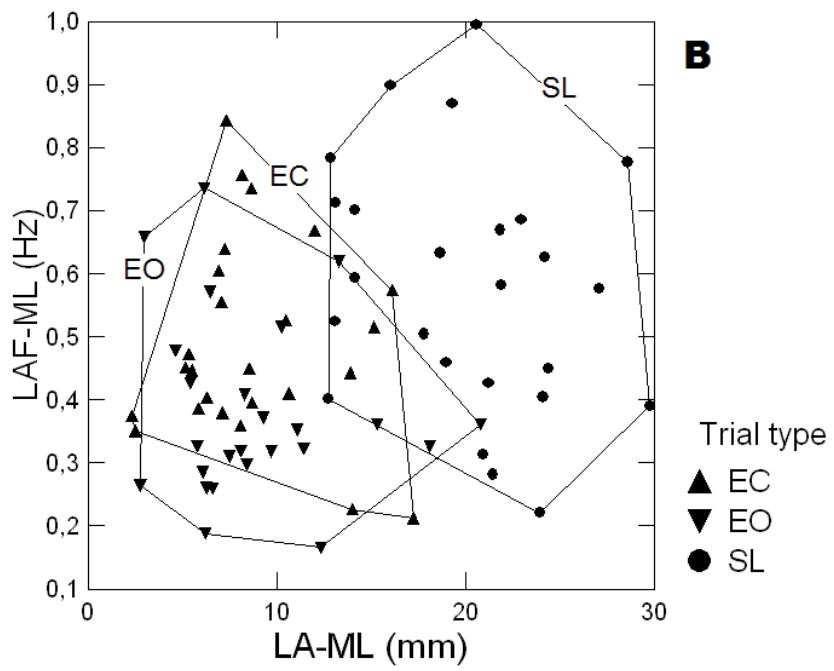

Fig. 5 Scatter plots of largest amplitude (LA) and largest amplitude frequency (LAF) in anteroposterior (AP, Fig. A) and mediolateral (ML, Fig. B) directions in eyes open bipedal stance (EO), eyes closed bipedal stance (EC) and eyes open single leg stance (SL)

\section{Conclusion}

On the basis of the results of both correlation analysis and variance analysis the following frequency-based CoP parameters of postural sway in both AP and ML directions are recommended to be used:

- Largest amplitude during balancing (LA)

- Frequency power ratios between low, medium and high frequency bands (LMR, MHR) 
- Spectral power ratio between AP and ML direction (SPR)

- Mean power frequency (MPF)

Many studies applied a wide variety of the studied frequency parameters on different neurologically or physiologically impaired patients, and they could come out with valuable results. However, our suggestion is that many of these parameters redundantly characterize postural stability, therefore a reduced number of non-correlating parameters should be enough to sufficiently characterize human balancing capability.

\section{Limitation of study}

The study examined only young healthy individuals in different stance types and gained no information about the behavior of parameters in physiologically or neurologically impaired patients. Time-frequency parameters were not applied in this study. The most important limitation of the study is that the testretest reliability of each parameter is not analyzed. The recommended reduced parameter set also needs to be tested using an intraclass correlation coefficient and a coefficient of variation.

$\begin{array}{ll}\text { Abbreviations } & \\ \text { AP } & \text { anteroposterior } \\ \text { BMI } & \text { body mass index } \\ \text { BW } & \text { bandwidth } \\ \text { CI } & \text { confidence interval } \\ \text { CoP } & \text { center of pressure } \\ \text { EC } & \text { bipedal stance with eyes closed } \\ \text { EO } & \text { bipedal stance with eyes open } \\ \text { FFT } & \text { Fast Fourier Transformation } \\ \text { I.S.P.G.R } & \text { International Society for Posture and Gait } \\ & \text { Research } \\ \text { LA } & \text { largest amplitude } \\ \text { LAF } & \text { largest amplitude freqnecy } \\ \text { LMR } & \text { low-medium frequency range power ratio } \\ \text { MDF } & \text { median frequency } \\ \text { MHR } & \text { medium-high frequency range power ratio } \\ \text { ML } & \text { mediolateral } \\ \text { MPF } & \text { mean power frequency } \\ \text { PSD } & \text { Power Spectral Density } \\ \text { SL } & \text { single leg stance with eyes open } \\ \text { SPR } & \text { AP-ML spectral power ratio } \\ \text { STFT } & \text { Short-time Fourier Transformation } \\ \end{array}$

\section{Acknowledgment}

This work was supported by the Hungarian Scientific Research Fund OTKA K083650.

\section{References}

[1] Terekhov, Y. "Stabilometry as a diagnostic tool in clinical medicine." Canadian Medical Association Journal. 115(7), pp. 631-633. 1976.

[2] Park, J-H., Youm, S., Jeon, Y., Park, S-H. "Development of a balance analysis system for early diagnosis of Parkinson's disease." International Journal of Industrial Ergonomics. 48, pp. 139-148. 2015.

DOI: 10.1016/j.ergon.2015.05.005

[3] Nagymate, G., Pethes, A., Szabo, G., Bejek, Z., Kiss, R. M. "The impact of the level of knee osteoarthritis on the postural stability." In: $32^{\text {nd }}$ Danubia-Adria Symposium on Advances in Experimental Mechanics, Zilina, Slovakia, Sep. 22.-25, 2015. pp. 26-27.

[4] Nagymate, G., Pethes, A., Szabo, G., Bejek, Z., Kiss, R. M. "Comparison of Postural Stability between Patients with Unilateral and Bilateral Knee Osteoarthritis." In: 11th Annual International Conference on Kinesiology and Exercise Sciences, Athens, Greece, Jul. 13-16, 2015. pp. 24-25.

[5] Nagy, E., Toth, K., Janositz, G., Horvath, G. "Postural control in athletes participating in an ironman triathlon." European Journal of Applied Physiology. 92(4-5), pp. 407-413. 2004. DOI: 10.1007/s00421-004-1157-7

[6] Oba, N., Sasagawa, S., Yamamot, A., Nakazawa, K. "Difference in Postural Control during Quiet Standing between Young Children and Adults: Assessment with Center of Mass Acceleration." PLOS ONE. 10(10), pp. 1-11. 2015. DOI: 10.1371/journal.pone.0140235

[7] Stensdotter, A. K., Bjerke, J., Djupsjöbacka, M. "Postural sway in singlelimb and bilateral quiet standing after unilateral total knee arthroplasty." Gait \& Posture. 41(3), pp. 769-773. 2015. DOI: 10.1016/j.gaitpost.2015.02.005

[8] Nilsson, G., Ageberg, E., Ekdahl, C., Eneroth, M. "Balance in singlelimb stance after surgically treated ankle fractures: a 14-month followup." BMC Musculoskeletal Disorders. 7(35), pp.1-8. 2006. DOI: 10.1186/1471-2474-7-35

[9] Lawson, T., Morrison, A., Blaxland, S., Wenman, M., Schmidt, C. G., Hunt, M. A. "Laboratory-based measurement of standing balance in individuals with knee osteoarthritis: a systematic review." Clinical Biomechanics. 30(4), pp. 330-342. 2015. DOI: 10.1016/j.clinbiomech.2015.02.011

[10] Nagymate, G., Kiss, R. M. "Replacing redundant stabilometry parameters with ratio and maximum deviation parameters." In: The $12^{\text {th }}$ IASTED International Conference on Biomedical Engineering. Innsbruck, Austria, Feb. 15-16, 2016. pp. 140-144. DOI: 10.2316/P.2016.832-022

[11] Carroll, J. P., Freedman, W. "Nonstationary properties of postural sway." Journal of Biomechanics. 26(4-5), pp. 409-16. 1993. DOI: 10.1016/0021-9290(93)90004-X

[12] Aoki, M., Tokita, T., Kuze, B., Mizuta, K., Ito, Y. "A characteristic pattern in the postural sway of unilateral vestibular impaired patients." Gait \& Posture. 40(3), pp. 435-440. 2014. DOI: 10.1016/j.gaitpost.2014.05.013

[13] Loughlin, P. J., Redfern, M. S, Furman, J. M. "Nonstationarities of postural sway." IEEE Engineering in Medicine and Biology Magazine. 22(2), pp. 69-75. 2003. DOI: 10.1109/MEMB.2003.1195699

[14] Schumann, T., Redfern, M. S., Furman, J. M., El-Jaroudi, A., Chaparro, L. F. "Time-frequency analysis of postural sway." Journal of Biomechanics. 28(5), pp. 603-607. 1995. DOI: 10.1016/0021-9290(94)00113-I

[15] Ferdjallah, M., Harris, G. F., Wertsch, J. J. "Instantaneous postural stability characterization using time-frequency analysis." Gait \& Posture. 10(2), pp. 129-134. 1999. DOI: 10.1016/S0966-6362(99)00023-5

[16] Chiaramello, E., Agostini, V., Balestra, G., Knaflitz, M. "Automatic classification of time-frequency plots applied to the center-of-pressure rotational components." In: 35th Annual International Conference of the IEEE Engineering in Medicine and Biology Society (EMBC). Osaka, Japan, July 3-7, 2013 pp. 4219-4422. DOI: 10.1109/EMBC.2013.6610476 
[17] Chiaramello, E., Knaflitz, M., Agostini, V. "Rotary spectra analysis applied to static stabilometry." In: 2011 Annual International Conference of the IEEE Engineering in Medicine and Biology Society (EMBC). Boston, MA, USA, 30 Aug.-3 Sept. 2011, pp. 4939-4952.

DOI: $10.1109 /$ IEMBS.2011.6091224

[18] Morales, C. J., Kolaczyk, E. D. "Wavelet-based multifractal analysis of human balance." Annals of Biomedical Engineering. 30(4), pp. 588-597. 2002. DOI: $10.1114 / 1.1478082$

[19] El-Jaroudi, A., Redfern, M. S., Chaparro, L. F., Furman, J. M. "The application of time-frequency methods to the analysis of postural sway." Proceedings of the IEEE. 84(9), pp. 1312-1318. 1996. DOI: 10.1109/5.535249

[20] Lyytinen, T., Liikavainio, T., Bragge, T., Hakkarainen, M., Karjalainen, P. A., Arokoski, J. P. A. "Postural control and thigh muscle activity in men with knee osteoarthritis." Journal of Electromyography and Kinesiology. 20(6), pp. 1066-1074. 2010. DOI: 10.1016/j.jelekin.2010.05.005

[21] Vieira, T. M. M., Oliveira, L. F., Nadal, J. "Estimation procedures affect the center of pressure frequency analysis." Brazilian Journal of Medical and Biological Research. 42(7), pp. 665-73. 2009.

DOI: 10.1590/S0100-879X2009000700012

[22] Nardone, A., Tarantola, J., Giordano, A., Schieppati, M. "Fatigue effects on body balance." Electroencephalography and Clinical Neurophysiology/Electromyography and Motor Control. 105(4), pp. 309-320. 1997. DOI: 10.1016/S0924-980X(97)00040-4

[23] Hasan, S. S., Robin, D. W., Szurkus, D. C., Ashmead, D. H., Peterson, S. W., Shiavi, R. G. "Simultaneous measurement of body center of pressure and center of gravity during upright stance. Part II: Amplitude and frequency data." Gait \& Posture. 4(1), pp. 11-20. 1996. DOI: 10.1016/0966-6362(95)01031-9

[24] Kanekar, N., Lee, Y. J., Aruin, A. S. "Frequency analysis approach to study balance control in individuals with multiple sclerosis." Journal of Neuroscience Methods. 222, pp. 91-96. 2014.

DOI: 10.1016/j.jneumeth.2013.10.020

[25] Schmid, M., Conforto, S., Camomilla, V., Cappozzo, A., D’Alessio, T. "The sensitivity of posturographic parameters to acquisition settings." Medical Engineering \& Physics. 24(9), pp. 623-631. 2002. DOI: 10.1016/S1350-4533(02)00046-2

[26] Scoppa, F., Capra, R., Gallamini, M., Shiffer, R. "Clinical stabilometry standardization: basic definitions - acquisition interval - sampling frequency." Gait \& Posture. 37(2), pp. 290-292. 2013.

DOI: 10.1016/j.gaitpost.2012.07.009
[27] van der Kooij, H., Campbell, A. D., Carpenter, M. G. "Sampling duration effects on centre of pressure summary measures." Gait \& Posture. 13(1), pp. 35-40. 2001. DOI: 10.1016/j.gaitpost.2011.02.025

[28] Ruhe, A., Fejer, R., Walker, B. "The test-retest reliability of centre of pressure measures in bipedal static task conditions - A systematic review of the literature." Gait \& Posture. 32(4), pp. 436-445. 2010.

DOI: 10.1016/j.gaitpost.2010.09.012

[29] Braun, B. J., Veith, N. T., Hell, R., Döbele, S., Roland, M., Rollmann, M., Holstein, J., Pohlemann, T. "Validation and reliability testing of a new, fully integrated gait analysis insole." Journal of Foot and Ankle Research. 8, p.54. 2015. DOI: 10.1186/s13047-015-0111-8

[30] Stewart, J. M., Clarke, D. "He’s dizzy when he stands up:an introduction to initial orthostatic hypotension." Journal of Pediatrics. 158(3), pp. 499-504. 2011. DOI: 10.1016/j.jpeds.2010.09.004

[31] Marks, R. J. "Introduction to Shannon Sampling and Interpolation Theory." Springer-Verlag, New York, 1991. DOI: 10.1007/978-1-4613-9708-3

[32] Bizid, R., Jully, J. L., Gonzalez, G., François, Y., Dupui, P., Paillard, T. "Effects of fatigue induced by neuromuscular electrical stimulation on postural control." Journal of Science and Medicine in Sport. 12(1), pp. 60-66. 2009. DOI: 10.1016/j.jsams.2007.10.006

[33] Oskoei, M. A., Hu, H. "Support vector machine based classification scheme for myoelectric control applied to upper limb." IEEE Transactions on Biomedical Engineering. 55(8), pp. 1956-1965. 2008. DOI: 10.1109/TBME.2008.919734

[34] Chan, Y. H. "Biostatistics 104: correlational analysis." Singapore Medical Journal. 44(12), pp. 614-619. 2003.

[35] Oliveira, L. F., Vieira, T. M. M., Macedo, A. R., Simpson, D. M., Nadal, J. "Postural sway changes during pregnancy: A descriptive study using stabilometry." European Journal of Obstetrics \& Gynecology and Reproductive Biology. 147(1), pp. 25-28. 2009.

DOI: 10.1016/j.ejogrb.2009.06.027

[36] Schmid, M., Conforto, S., Lopez, L., Renzi, P., D’Alessio, T. "The development of postural strategies in children: a factorial design study." Journal of NeuroEngineering and Rehabilitation. 29(2), 2005.

DOI: 10.1186/1743-0003-2-29

[37] Pasman, E. P., Murnaghan, C. D., Bloem, B. R., Carpenter, M. G. "Balance problems with Parkinson's disease: are they anxiety-dependent?." Neuroscience. 177, pp. 283-291. 2011.

DOI: $10.1016 /$ j.neuroscience.2010.12.050 